\title{
MANERAS DE COMER HOY Comprender la modernidad alimentaria desde y más allá de las normas
}

\author{
MABEL GRACIA ARNAIZ \\ Universitat Rovira i Virgili
}

\section{PALABRAS CLAVE ADICIONALES}

Medicalización, Desestructuración alimentaria,

Normas y prácticas alimentarias, Macdonalización.

\section{ADDITIONAL KEYWORDS}

Medicalization, Food Desestructuration, Food

Rules and Practices, Mcdonalization.

RESUMEN. Numerosos estudios sobre las maneras de comer en las sociedades industrializadas han tenido como finalidad analizar hacia donde va la alimentación. No obstante, la mayoría de estos estudios han privilegiado una orientación económica (interesándose por las compras y la evolución del gasto) o nutricional (interesándose por la evolución de la dieta). Desde estas perspectivas, las prácticas actuales, incluidas bajo el paraguas de la denominada modernidad alimentaria, son objeto de inquietudes facultativas y de incertidumbres socio-económicas sobre las que se debe actuar e intervenir. Algunas de las hipótesis formuladas desde la antropología y la sociología aceptando la desestructuración de las maneras de comer contemporáneas han sido utilizadas para reafirmar, en buena medida, tanto la intervención médico-nutricional como el negocio de la industria alimentaria, mientras que otras hipótesis han criticado abiertamente la aceptación de esta supuesta degradación. En el centro del debate está la necesidad de diseñar estudios que metodológicamente puedan caracterizar las maneras de comer y dar cuenta de los factores que verdaderamente determinan las prácticas alimentarias, su transformación o su inmutabilidad.

\begin{abstract}
Numerous studies on the eating habits of industrialized societies have focused on analyzing the direction in which diet is moving. Most of these studies have been carried out either from an economic point of view (discussing purchases and changes in expenditure) or from a nutritional point of view (describing changes in diet). From these perspectives, current practices, which fall under the umbrella of the so-called dietary modernity, are the object of medical are the objet of medical concerns and socioeconomic uncertainties that must be addressed because "people in modern societies do not know how to eat". Some of the hypotheses formulated from the anthropological and sociological standpoints that have accepted the destructuring of contemporary eating habits have been used to support to a large extent both medical and nutritional intervention and the business of the food industry. Other hypotheses, on the other hand, have openly criticized the acceptance of this supposed destructuring. Central to the debate is the need to design studies that can characterize eating habits methodologically, highlight which factors are really behind these habits, and reveal whether these habits are indeed changing.
\end{abstract}

E-mail: mabel.gracia@urv.net

\section{Revista Internacional de Sociología (RIS)}

Tercera Época, Nº40, Enero-Abril, 2005, pp. 159-182. 


\section{INTRODUCCIÓN}

Las investigaciones sobre alimentación realizadas desde ámbitos institucionales rara vez se han revestido de un carácter inocente o han estado simplemente motivadas por la inquietud de conocimiento (De Garine 2002: 10-11). Así, por ejemplo, los trabajos pioneros de las antropólogas Richards (1932 y 1939) y Mead (1943) se inscriben dentro de las preocupaciones que las potencias coloniales o los propios Estados tenían para asegurar una "alimentación equilibrada" (léase también medicalizada) a las poblaciones dominadas, inmigrantes o autóctonas. En un contexto en el que los hábitos alimentarios experimentaban transformaciones notables, la inquietud provenía desde las instituciones de salud pública, pero también de la necesidad de conservar una mano de obra capaz de trabajar eficazmente y de adaptarse lo mejor posible a situaciones críticas de escasez o, simplemente, de cambio ante la oferta de alimentos. Motivados también por una inquietud de aplicación práctica, durante la Segunda Guerra Mundial, Guthe y Mead crearon en EEUU el Comité para el Estudio de los Hábitos Alimentarios, destinado a facilitar ayuda alimentaria a los países que poseían una cultura distinta de la estadounidense y, eventualmente, a permitir al personal militar que se encontraba allí desplazado subsistir sin riesgo alguno.

Al final de la Segunda Guerra Mundial se articularon, por iniciativa de la F.A.O y de la O.M.S, una serie de programas de ayuda alimentaria, nutrición aplicada y educación sanitaria, destinados a mejorar el nivel nutricional de diferentes grupos sociales, en particular de los grupos llamados "vulnerables" en los países del Tercer Mundo. Entre sus objetivos principales estaba averiguar cómo inducir a las personas a alimentarse mejor (ya fuera aprovechando lo que tenían a su alrededor, ya fuera importando productos manufacturados) o cómo calcular y establecer una ración alimenticia satisfactoria para las diferentes comunidades humanas. Desde entonces, la elaboración de patrones alimentarios, de estándares dietéticos y de recomendaciones nutricionales ha ido adquiriendo una relevancia central en el diseño de las políticas sanitarias internacionales, regionales o locales, destinadas no ya a los países en vías de desarrollo donde el fenómeno del hambre y sus negativas consecuencias es recurrente, sino a los países de todo el planeta y afectando a los grupos sociales más diversos (escolares, militares, prisioneros, enfermos, astronáutas, etc.).

En las sociedades industrializadas, donde la salud pública, el consumo y la seguridad alimentaria constituyen ámbitos de interés prioritario, una buena parte de los estudios que se están llevando a cabo tiene como finalidad principal señalar las tendencias alimentarias, preguntándose hacia donde va nuestra alimentación. Ello ha implicado la proliferación de documentos e informes de base estadística cuyo objetivo principal ha consistido en analizar la evolución del consumo alimentario y las actitudes de los distintos grupos sociales ante las nuevas ofertas de alimentos. Ahora bien, todos estos trabajos se han orientado principalmente desde una perspectiva económica (estudiando únicamente la evolución del gasto y 
las compras) o nutricional (estudiando sólo la evolución de la dieta en términos de nutrientes y composición), dejando en un segundo término el análisis de los factores y circunstancias socioculturales que condicionan el comportamiento alimentario, los cuales podrían explicar, en primera instancia, el porqué de tales tendencias y la lógica que subyace tras las elecciones alimentarias.

Las maneras actuales de comer, que normalmente se incluyen bajo el paraguas de la denominada modernidad alimentaria, constituyen el objeto de inquietudes facultativas e incertidumbres económicas y sociales (demasiadas grasas, exceso de azúcares, excesiva desestructuración, disminución relativa del gasto en ciertos alimentos...): jlas personas de las sociedades modernas no saben comer, son enfermos de la abundancia y de la civilización! La tentación de decodificar tales prácticas como la degradación de un orden alimentario inicial - también de un orden social - y de buscar la restauración de un estándar "tradicional" — supuestamente más racional y saludable - es extraordinaria. Desde esta perspectiva, el modelo alimentario actual se caracterizaría por la toma de decisiones individuales, a menudo compulsivas y anómicas, que promueven en cualquier caso una mayor demanda de consejos nutricionales en materia de alimentación.

Por otro lado, estas inquietudes parecen contradecirse con la importancia que las personas otorgan a su alimentación. En España, por poner un ejemplo específico y de acuerdo con numerosas estadísticas manejadas en los últimos años (MAPA, ICE, EPF, OCDE...), la salud aparece como una de las preocupaciones fundamentales de nuestra sociedad; se ha convertido en un valor cultural de primer orden, constituyéndose en un argumento fundamental del consumo de todo tipo de bienes y servicios. La alimentación es el ejemplo más evidente de esta evolución: "llevar una buena alimentación" es, para el $91 \%$ de la población española, el factor más relevante para conseguir una buena salud (IV Foro Internacional de la Alimentación, 2002).

No obstante, esta mayor preocupación por la salud contrasta con la circunstancia de que los alimentos cuyo consumo ha ido en aumento en las últimas décadas no son los más saludables desde un punto de vista nutricional, mientras que de forma paralela ha ido disminuyendo el consumo de aquellos otros que supuestamente sí lo son. Es el caso, por un lado, del incremento relativo de carnes y derivados, de la pastelería industrial o de ciertos platos precocinados $\mathrm{y}$, por otro, de la disminución de cereales o legumbres ¿Qué está sucediendo entonces? ¿Preocupa realmente la salud, prima la falta de tiempo y la comodidad en el consumo de ciertos alimentos o se eligen sólo los que proporcionan placer a los sentidos? De nuevo, nos encontramos con la misma cuestión: si bien se conocen ciertos datos sobre el consumo de alimentos y el aumento de la incidencia de ciertas enfermedades, se desconoce de una forma absoluta cuáles son las causas que guían las elecciones alimentarias y el modo en que están determinadas por factores socioculturales. Y la salud, aunque para la población española es una motivación importante de las elecciones alimentarias, no es ni la única ni, a menudo, la principal (Contreras y Gracia, 2004: 170). 


\section{LA MEDICALIZACIÓN DEL COMPORTAMIENTO ALIMENTARIO}

Hay que situar la actual relevancia social concedida a la salud y al seguimiento de las normas dietéticas, en el paulatino proceso de medicalización de la alimentación (Conrad, 1992; Poulain, 2002a) que, iniciado en el siglo XVIII, ha supuesto la sustitución de una buena parte de los motivos simbólicos, económicos o hedonistas sobre los cuales se articulaban las elecciones alimentarias por otros motivos de orden médico. En esa época, el físico George Cheyne, autor de la célebre obra The English Malady (1733), adoptó la metáfora mecánica del cuerpo según la cual la comida constituía el combustible que abastecía la máquina humana. Su objetivo era discutir sobre la importancia de los regímenes dietéticos, considerando que la "dieta rica" (basada en comidas y bebidas exóticas, abundantes y caras) constituía el origen de numerosas enfermedades entre la clase alta inglesa, por definición según el autor, inactiva y lujuriosa.

A partir del XIX, la prescripción de límites al consumo excesivo de alimentos no sólo se llevó a cabo en nombre de la "buena" salud, sino también de la delicadeza social y los buenos modales, especialmente entre las mujeres de las clases altas (Veblen, 1899; Bourdieu, 1988; Mennell,1985). En esos momentos, y en particular en las áreas de influencia protestante, las recomendaciones dietéticas formaron parte de una moral religiosa con implicaciones individuales, según la cual el cuerpo era una parte del reclamo ético: los desórdenes corporales debían resolverse con disciplina, autocontrol y vida sobria, evitando el exceso y el gasto innecesario. La dieta, en este caso entendida ya como un patrón de evitación o restricción, producía, entre otras virtudes, templanza, control de las pasiones, estabilidad mental y salud.

Según la hipótesis de Turner (1999), se produjo una paulatina afinidad entre la gestión facultativa de la dieta y la expansión del capitalismo, de tal forma que la nutrición, disciplinando el cuerpo y procurando una fuerza de trabajo fuerte y sana, se configuró en una ciencia altamente compatible con el espíritu del modo de producción capitalista. A finales del XIX, la dieta de todos los miembros de la sociedad se había construido como un problema biosocial, de forma que la gente tenía que vivir de acuerdo con las recomendaciones nutricionales velando por su salud corporal, especialmente las clases trabajadoras en cuanto que sus cuerpos eran las máquinas que les permitían ganarse el pan y, a la vez, contribuir a reproducir socialmente el sistema. Fue así como, de ahí en adelante, los discursos nutricionales se van a convertir progresivamente en "fábricas" de moral cuyo objetivo principal será instaurar la normalidad dietética y, en definitiva, la normalidad social (Coveney, 1999). El recurso de la teoría del victim blaming (Ryan, 1971; Crawford, 1977) a lo largo del siglo pasado, según la cual hay que reducir los comportamientos de riesgo atacándolos en razón de sus dimensiones morales, es decir, estigmatizándolos y transformando a las víctimas en culpables ("si usted está enfermo es porque no se ha portado bien..."), constituye otro paso adelante en el proceso de medicalización de la alimentación. 
En la actualidad se practica, sin embargo, lo que se ha demoninado la "comunicación sanitaria positiva"; es decir, se busca una posición menos culpabilizadora del sujeto a partir de la educación y la promoción para la salud. Este modelo de acción, cuyos contenidos han sido considerablemente interiorizados por la población en forma de "normas", sigue descansando, no obstante, en la idea de que los cambios en los comportamientos alimentarios descansan sobre dos factores biologicistas (Adam y Herzlich, 1994): la percepción de una amenaza para la salud física y la percepción (o la creencia) de que la adopción de un comportamiento particular puede reducir esa amenaza.

Las políticas de educación nutricional, en un claro intento por concretar sus ambiciones educativas en materia de nutrición y controlar así una buena parte de la vida cotidiana a través de las prácticas alimentarias, recurren al principio de que para modificar los hábitos alimentarios es suficiente con proporcionar a la población información de base científica. De este modo, las personas, siguiendo el consejo facultativo razonado, adoptan los comportamientos alimentarios mejor adaptados y convenientes para su salud. Dicha información, articulada en forma de campañas sanitarias, tiene como objetivo reeducar los comportamientos y reducir así las conductas de riesgos asociados. Desde esta perspectiva, se trataría de eliminar las motivaciones "no-racionales" que guían las preferencias humanas a través de la intervención médica y la puesta al día de la prescripción de dietas. La constatación más significativa es que tales propuestas dietéticas están sujetas a variaciones que ponen en cuestionamiento, a su vez, la oportunidad de un dogma de base científica. Se podría sostener, incluso, que la medicina y la nutrición también generan modas dietéticas, en el sentido de que animan a la población a seguir prácticas muy específicas durante cortos periodos de tiempo (consumir aceite de girasol, vísceras y despojos, pescados blancos, lácteos, carnes rojas, etc.).

Por esa razón, queremos detenernos ahora en este punto. Algunas propuestas facultativas, como hemos señalado en otro texto anterior (Gracia 1996), han alterado comportamientos alimentarios con el objetivo de mejorar la salud colectiva de la población cuestionando la bondad de consumos. Es el caso del cuestionamiento del azúcar, la sal o las grasas saturadas. En una obra clásica, Tannahill (1988), haciendo referencia a la sociedad británica, menciona alguna de las apreciaciones significativas que la ciencia médica ha ido manteniendo y modificando durante las últimas décadas respecto a dichos productos:

- Azúcar: En 1968, la Asociación de Investigación Biológica anunciaba que se había encontrado una relación entre el consumo de azúcar y la aparición de enfermedades cardiovasculares. En 1983, el Real Colegio de Físicos de Londres recomendaba que se ingiriera la mitad de la ingesta nacional de azúcar porque proporcionaba muchas calorías sin ningún valor nutritivo. Unos años más tarde, en 1986, se publicaron los resultados de un amplio estudio sobre esta sustancia. El trabajo médico de la Food and Drug Administration puntualizó que el 
azúcar sólo era peligrosa en su contribución a la caída de los dientes y que no se podía establecer ningún vínculo categórico por falta de evidencias entre el azúcar y la obesidad, la diabetes, la alta presión arterial o las enfermedades del corazón.

- Sal: Entre 1972-75 un estudio realizado en dos ciudades de California concluía que los individuos que durante ese período redujeron el consumo de sal en un $30 \%$, su presión arterial registró una caída del $6.5 \%$. Una década más tarde, se afirmaba que la hipertensión estaba vinculada con la mitad de las muertes ocurridas anualmente en los Estados Unidos. En consecuencia con estos resultados, Japón, que registraba una ingesta media de sal altísima, aparecía como el país con más posibilidades de ser proclamado el de mayor ratio de hipertensión del mundo. Sin embargo, una investigación realizada por la Organización Mundial de Salud en 1986 presentó a Japón como el país con el índice de enfermedades del corazón más bajo del mundo.

- Grasas saturadas: Varias investigaciones realizadas entre los años 60 y 70 apoyaban la creencia de que el consumo de grasas saturadas aumentaba peligrosamente el nivel de colesterol, incidiendo de forma negativa en las arterias coronarias. Más tarde, en 1981 se descubrió que en el cáncer de mama, una enfermedad hasta entonces asociada a dietas ricas en carnes y grasas, el estreñimiento aparecía como un factor de correlación más importante que la propia ingesta de grasas. Cuatro años después, otra investigación demostró que Francia, el país con el consumo más alto de grasas en la Unión Europea, registraba uno de los índices más bajos de enfermedades cardiovasculares.

Con toda esta casuística científica, Tannahill advierte de los riesgos del dogma dietético derivados de la actitud empiricista adoptada por una parte significativa de los profesionales sanitarios. En 1986, la British Medical Association afirmaba que el azúcar, la sal y las grasas saturadas eran potencialmente perjudiciales para el organismo, aun cuando su relación con estados precarios de salud no había sido todavía probada. Estas consideraciones, que han podido afectar a alimentos potencialmente saludables, han sido sancionadas por el paradigma médico. Así pues, la información científica también favorece el eclecticismo, y aunque los educadores de la salud parecen utilizar siempre argumentos convincentes, lo cierto es que sus datos, como destaca Tannahill, están muy lejos de poder considerarse concluyentes. Deschamps y Spykerrelle (1988) también suscriben la misma idea, advirtiendo que los consejos dados por los médicos y nutricionistas varían de un período a otro, de un manual a otro. Hay que entender que la ciencia es dinámica y los resultados de las investigaciones cambian. Ello no impide, sin embargo, que ciertos valores difundidos por el propio saber médico-nutricional sean interiorizados por la población en forma de normas dietéticas confusas sabiendo que, ante las evidencias, tampoco esta información está exenta de contradicciones. 
Por otro lado, la medicalización de la alimentación no es una cuestión únicamente de facultativos, sino que es el resultado de interacciones complejas entre los diferentes actores sociales que, de un modo $u$ otro, esperan canalizar sus intereses. Los industriales agroalimentarios tienden a utilizar también el argumento de la salud con fines promocionales en sus generosas inversiones publicitarias y a convertir sus productos en "alimentos funcionales" o "alicamentos", reivindicando las supuestas cualidades dietéticas de una buena parte de los nuevos artículos lanzados al mercado porque dicho reclamo les es rentable económicamente.

Esta concepción nutricional de la alimentación no toma en cuenta, sin embargo, los constreñimientos materiales que condicionan a las personas ni por supuesto el valor de la sociabilidad alimentaria: cuidar de la salud, vigilar la comida, hacer dieta, por ejemplo, dependen de cuestiones tales como los recursos disponibles y de condicionantes vinculadas con el trabajo, la renta o la vida familiar y social. Dejar de beber o comer un alimento o bebida, como dejar de fumar o de conducir deprisa, puede constituir una decisión individual fundada sobre una información objetiva o sobre una norma, pero es necesario conocer la dificultad que conlleva introducir ciertos cambios, ya que la mayor parte de los comportamientos humanos están modelados por la cultura a la que pertenecen los individuos. Si realmente se quiere comprender cómo los saberes, las representaciones y los discursos alimentarios toman sentido para la acción, conviene relacionarlos, por un lado, con los constreñimientos cotidianos de la vida de la gente y, por otro, con las características de sus relaciones sociales. Modificar los hábitos alimentarios, aun con ánimos bienintencionados de "reformar" la conducta para alcanzar una vida más saludable, es mucho más complicado de lo que se puede pensar, tal como vienen demostrando las ciencias sociales desde hace ya bastantes décadas (Richards, 1932 y 1939; Lewin, 1943; Mead, 1943).

Por otro lado, numerosos estudios indican que ni la acumulación, ni siquiera la comprensión, de los conocimientos nutricionales son necesariamente suficientes para hacer cambiar los hábitos alimentarios de las personas. Hace dos décadas, Thomas (1980) analizó la relación entre, un lado, el conocimiento sobre los alimentos y la nutrición y, otro, las elecciones alimentarias, para demostrar que no siempre un mayor conocimiento nutricional por parte de la población tiene que ver con conductas alimentarias más óptimas. La autora hizo una revisión de las investigaciones hechas en Gran Bretaña entre los años 60 y 80 sobre el grado de conocimiento nutricional de las amas de casa. Algunos trabajos de finales de los 60 evidenciaban, en primer lugar, que ese conocimiento dependía de ciertas variables. Así, las amas de casa más jóvenes y con un estatus socioeconómico superior mostraban un conocimiento nutricional más detallado. En la década siguiente, las investigaciones señalaban que se producía un conocimiento paulatino sobre un mayor número de tipos de nutrientes, así como de términos específicos, tales como el colesterol o las grasas polisaturadas. Ahora bien, Thomas advertía que estar familiarizado con la terminología nutricional, incluso incorporarla en el lenguaje 
RIS

REVISTA INTERNACIONAL DE SOCIOLOCIA

No 40 , ENERO-ABRIL, 2005

MABEL GRACIA ARNAIZ

cotidiano, podía ser una interpretación muy limitada de lo que ha de entenderse por conocimiento. Normalmente, esta idea se ha relacionado con aspectos vinculados al tipo de nutrientes que componen los alimentos o las funciones que éstos tienen. Los estudios hechos en dicha línea destacaban una evolución positiva en el conocimiento de la composición de vegetales y carbohidratos, más que de proteínas o, incluso, de las funciones de algunos nutrientes. Por ejemplo, el calcio se asocia habitualmente con la osificación o la dentadura y el hierro como un mineral necesario para la sangre.

Sin embargo, Thomas cuestionaba que estos valores fueran los indicadores más idóneos para medir el conocimiento nutricional de la población en cuanto que los datos resultantes de esos estudios enmascaraban cierta superficialidad. De hecho, ella observó que los resultados de los tests nutricionales no coincidían con los datos obtenidos al analizar las prácticas alimentarias que las madres seguían cuando alimentaban a sus hijos, siempre más positivas desde este punto de vista. Por ello, concluyó que era más interesante relacionar el conocimiento nutricional con las propias elecciones alimentarias. Una actitud importante registrada en el $69 \%$ de las mujeres entrevistadas en un trabajo realizado por Sims a mediados de los setenta coincidía con la siguiente afirmación: cuando el ama de casa compra la comida está más preocupada pensando qué comerá su familia que descubriendo qué es lo mejor para ella. Así pues, los estudios de esta década siguen confirmando que el hecho de que el conocimiento nutricional varíe, incluso se amplíe, no va acompañado de un cambio hacia conductas alimentarias más saludables.

Algunos de estos trabajos especifican, incluso, que determinados programas de educación nutricional apenas tienen efecto en los hábitos de las personas a quienes se dirigen. Herbert (1990) advierte el mismo nivel de desconocimiento nutricional y de nociones dietéticas supuestamente erróneas en la población estadounidense. Según los resultados de una encuesta llevada a cabo en 1989 entre mil adultos, el $68 \%$ de los entrevistados no sabía que sólo hubiera colesterol en los productos de origen animal, aunque éste constituyera, al igual que en los estudios citados por Thomas, uno de los términos más referidos y preocupantes para la población. Una relativa confusión también se detectó a la hora de clasificar los alimentos en grupos. Mientras que el $61 \%$ consideraba que el brécol era una legumbre en vez de un vegetal, el $41 \%$ creía que el ketchup, un condimento, era un vegetal. Lo mismo sucedía cuando se trataba de relacionar los conocimientos nutricionales con el seguimiento de régimenes para controlar el peso. Así, un porcentaje elevado de personas pensaba que la pasta y las patatas engordaban más que los bistecs de carne. En una línea semejante, es decir, tratando de evaluar el tipo y grado de conocimientos de carácter nutricional, el estudio llevado a cabo en España, ¿Adónde va nuestra alimentación? (2002), apuntaba que el $41 \%$ de las personas entrevistadas no pudieron citar ningún mineral recomendado para obtener buena salud y que a la pregunta "qué miden las calorías" el $79 \%$ de los entrevistados no contestó nada. 
Nos encontramos, pues, con otra aparente contradicción. Por un lado, parece ser que haber incorporado el lenguaje nutricional cuando se habla de alimentos y comida o, incluso, disponer de más conocimientos nutricionales, no ha implicado necesariamente un cambio "positivo" en las prácticas alimentarias, mientras que, por otro, las personas que hacen las elecciones más acertadas desde el punto de vista de la salud son las que, también según estos estudios, disponen de una mayor información nutricional. Thomas ya señaló que la adopción de nuevas prácticas acordes con este tipo de conocimientos especializados no es sistemática, sino que tiene que ver con factores de orden social y motivacional antes que sanitario. Por ejemplo, si hay niños en la familia, las personas responsables de la alimentación cotidiana parecen más dispuestas a modificar las prácticas alimentarias que si no los hay. El aprendizaje y la aplicación de los programas educacionales también dependen del grado de implicación que envuelve a las personas y de sus posibilidades prácticas de llevarlos a cabo. Por esa razón, Thomas sugería con acierto que, hasta que no se tenga en cuenta este tipo de relaciones, todos los intentos de mejorar las elecciones alimentarias con fines nutricionales están destinadas al fracaso.

En la misma línea, Poulain (2002b) muestra en un estudio reciente cómo los franceses, al describir sus prácticas alimentarias y argumentar el porqué de ciertas elecciones, se alejan de las prescripciones nutricionales dependiendo de las circunstancias contextuales, mientras que priman las dimensiones culturales, basadas a menudo en la conveniencia, en el placer o la identidad. En este sentido, la población francesa tiene un conocimiento simultáneo de los alimentos "buenos", que son óptimos para la salud, y de los alimentos que debe controlar para mantener una buena salud o no engordar ("malos"). Sin embargo, sus clasificaciones de los "alimentos esenciales" apenas coinciden con la de los "alimentos más adecuados para la salud". Confirmando este desfase, el estudio socio-antropológico realizado por Contreras y Gracia (2004: 173-4) indica, para el caso español, un contraste importante entre las ideas que sustentan las elecciones alimentarias los días de "diario" y las de los "fines de semana": las personas que dicen preocuparse por la dieta durante la semana en términos de salud dicen también que "cuando llega el domingo, hacemos un poco de extra", de tal manera que se subordina la "bondad" o el "control" que se guarda durante la semana, representada sobre todo por la verdura y la fruta, al "extra" o al "capricho", representado por "el arroz y todo lo que entra", los dulces, los vinos, los helados, etc: Por otra parte, la comodidad y la salud son los objetivos perseguidos en los días ordinarios. La comodidad, por el constreñimiento del tiempo; la salud, porque se trata de la mayoría de los días: "yo de lunes a viernes intento no pasarme en nada, ni un capricho". Sin embargo, el acuerdo es unánime en que los días festivos o los fines de semana se relaja la preocupación por la salud o la preocupación por una dieta "más sana" y aparece un mayor interés por una comida considerada más "placentera". Dichos resultados confirman, de nuevo, que la salud no es el único objetivo ni la única función de la alimentación. 


\section{HIPÓTESIS SOBRE LA NATURALEZA DEL ACTUAL MODELO ALIMENTARIO}

Algunas de las hipótesis que se han barajado desde la antropología y la sociología de la alimentación han podido consolidar, en cierta medida, la intervención médiconutricional en los términos descritos anteriormente, aunque otras han cuestionado el alcance de la medicalización de la alimentación cotidiana, afirmando que el modelo alimentario moderno no sólo es racional culturalmente hablando, sino que presenta un "estado de salud" inmejorable respecto a periodos anteriores.

A principios de los años 80 , diferentes estudios constataban una cierta desestructuración de la alimentación en los países más industrializados (Douglas, [1979]1995; Fischler, 1979; Herpin, 1988), siendo sus principales síntomas la desconcentración, la desocialización, la desinstitucionalización, la desimplantación horaria o la desritualización alimentaria. En esos contextos, la regularidad, la composición y el curso de las comidas parece que habían evolucionado de forma muy rápida durante los últimos treinta años. Como indicadores que apuntarían en esa dirección se consideraba el aumento del número de ingestas realizadas a lo largo del día, la supresión de platos en los menús, el incremento de comidas hechas en solitario o fuera de casa, la simplificación de las cenas o la desregulación de los horarios.

Según Fischler (1995), resulta paradójico que, en la sociedad de la abundancia, un problema cotidiano pueda ser el preguntarse qué cómo hoy y cómo. Para este autor, el comensal urbano se convierte en un individuo mucho más autónomo en sus elecciones y rebasa sus limitaciones sociales hacia conductas individuales: los tiempos, ritos y compañías se imponen cada vez más con menos formalismos. La comida, de acuerdo con Fischler, se ha vuelto tan problemática, está tan lejos de ser sencilla, que hoy nos preocupamos por enseñar a los niños a comer y nos hemos inventado para ello "la educación nutricional". En efecto, el comensal contemporáneo ha perdido su capacidad para adoptar decisiones óptimas porque apenas cuenta con informaciones coherentes, en tanto que la cacofonía de criterios propuestos culturalmente (que van desde los consejos médicos a los publicitarios, pasando por un sinfin de alternativas dispares entre sí) lo único que ha logrado es instaurar una incertidumbre perpetua. La sociedad urbana es una sociedad gastro-anómica: las comidas familiares disminuyen, el tiempo dedicado a comer cada vez es menor, se come en cualquier parte y cualquier cosa. Estamos, en consecuencia, ante una "crisis de la civilización industrial" o ante la "locura de la cultura" que se manifiesta claramente en el aumento del snacking (picoteo) y del vagabundeo alimentario.

Thay quienes atribuyen esta subjetivación de las prácticas alimentarias al descenso de las presiones ejercidas por las categorias sociales de pertenencia (Giddens, 1991 y 1996; Bauman, 2001; Beck, 2001), lo que se traduciría en un debilitamiento de los grandes determinismos sociales, principalmente de las clases, que pesan sobre los individuos y sus prácticas de consumo. Desde esta perspectiva, 
se remarca que la gente puede elegir sus propios packs de consumo dentro de una amplia gama de posibilidades. El argúmento de la diversidad alimentaria, referido como una alimentación quasi postfordista en términos de variedad, propugna la idea de que el nicho de consumo es voluntario y resultado de un sistema capitalista que tiende a una producción más flexible (Warde 1997).

La naturaleza de la modernidad alimentaria es, sin embargo, ambivalente, en tanto que una parte de las tendencias actuales señalan en una dirección contraria: la aplicación de los principios de la macdonalización (eficacia, predictibilidad, calculabilidad y control) a todos los ámbitos de la vida social y en diferentes países del mundo confirmaría una homogenización de las prácticas $\mathrm{y}$, en definitiva, una estandarización de los hábitos alimentarios (Ritzer, 1993 y 2001; Fischler, 1996; Ariès, 1997 a, 1997 b). En general, se suele hablar de homogeneización e internacionalización alimentaria en dos sentidos (Gracia 1998). Por una parte, en la falta de variedad nutricional de las dietas, lo que supone que, contraviniendo nuestra naturaleza omnívora, reducimos y desestimamos las posibilidades de obtener energía a partir del consumo variado de alimentos y, en consecuencia, acabamos registrando excesos o carencias de orden nutricional; en los países industrializados, como señalábamos antes, éste es un aspecto que preocupa especialmente a los técnicos sanitarios y a las políticas alimentarias. Por otra parte, se suele hablar de homogeneización por la similitud de consumos entre los diferentes países a partir de la disminución de las variedades locales. Dejando aparte el hecho de que, en efecto, bastantes artículos locales han cesado su producción durante estos años, tal afirmación pasa por alto, sin embargo, que la homogeneización tiene un largo pasado histórico que se refleja en las monotonías locales. Por ejemplo, las cocinas regionales francesas no se consolidaron hasta el siglo XIX, de manera que tampoco son tan antiguas como a veces se pretende mostrar. En la Edad Media, amplias regiones participaban de una alimentación parecida; estudios hechos sobre la cocina provenzal del siglo XIV muestran que incluso las diferenciaciones entre cocina aristocrática y popular no tenían excesiva relevancia (Goody 1989).

Relacionada con la tendencia homogeneizadora de los consumos, también se ha hablado de la mundialización o internacionalización de la cocina. Es cierto que no se deben subestimar las diferencias regionales existentes ni los movimientos de autodefensa por conservarlas, pero la similitud de los productos expuestos en los supermercados de cualquier país, las bebidas ofrecidas en todos los aeropuertos o las comidas servidas en los hoteles internacionales son, según Goody (1989) y Fischler (1995), evidencias de la uniformidad de gustos vinculada con el carácter industrial y el nuevo orden mundial. Dicha mundialización de la cocina viene favorecida, a su vez, por dos procesos distintos. Por un lado, por el auge de la migración de las cocinas, iniciada ya hace décadas (aunque frecuentemente tengan poco que ver con las cocinas de origen) y, por otro, por el desarrollo de una cocina propiamente internacional (por ejemplo, en las redes hoteleras predomina un tipo de comida internacional de base franco-italiana). 
RIS

REVISTA INTERNACIONAL DE SOCIOLOGIA

№ 40, ENERO-ABRIL, 2005

No obstante, también es necesario señalar, de acuerdo con Poulain (2002: 20-25), que los particularismos nacionales y regionales no desaparecen tan rápidamente como algunos han sugerido recientemente. Ciertamente, McDonald's, aun siendo el primer restaurante mundial y la imagen misma de la homogeneización, se ha visto obligado a tener en cuenta este tipo de particularidades a la hora de introducirse en las diferentes culturas alimentarias del planeta, estableciendo estrategias de microdiversificación para adaptarse a los gustos de los mercados locales. La estrategia inicial de esta cadena de restaurantes rápidos consideraba que su oferta - es decir, la gama de productos resultantes de una organización semejante a la utilizada en la fabricación seriada de automóviles - era inmodificable, poniéndose como objetivo superar los obstáculos de su aceptación a través del manejo de la comunicación y el marketing. Sin embargo, ante la resistencia de los mercados, poco a poco fue introduciendo una serie de modificaciones en la oferta para adaptarla a los hábitos locales. En Francia, por ejemplo, en los restaurantes McDonald's se sirve cerveza, mientras que en los EEUU no hay bebidas alcohólicas. En Francia, Holanda, Bélgica la mayonesa acompaña a los fritos, mientras que en los EEUU el ketchup goza de una supremacía indiscutible. En sus inicios, esta cadena producía un café ligero tipo americano que no gustaba nada a italianos o españoles; hoy, las tecnologías han sido adaptadas para ofrecer tipos de café también más parecidos a los gustos locales. En este sentido, pues, los particularismos locales todavía son bastante fuertes. Junto a los Big Mac empiezan a aparecer "platos étnicos" que tratan de dar un contenido local dentro de una oferta global. En Inglaterra, por ejemplo, McDonald's ha intentado asimilar la comida hindú con su McSpicy Burger y McChicken Korma y Vegetable Samosa.

La internacionalización de las cocinas contrasta, además, con los esfuerżos de los chefs de la restauración, de los gastrónomos o incluso de las autoridades locales, por recuperar o inventar cocinas nuevas, tradicionales, regionales o nacionales; unas cocinas que, analizadas en su conjunto, tienen poco que ver con las comidas cotidianas de la mayoría de la población. No obstante, el empeño por destacar la supuesta diversidad y la peculiaridad culinaria de cada cultura o país es significativo y es una reacción lógica a la deslocalización que han sufrido los alimentos modernos al ser separados de su contexto geográfico y de los constreñimientos climáticos a los que tradicionalmente estaban asociados. De forma simultánea, el miedo a la estandarización está siendo utilizado por diferentes sectores (restauradores, políticos, asociaciones culturales) para reivindicar el mantenimiento o la restitución de las cocinas regionales y autóctonas.

Así pues, no todos los estudiosos estarían de acuerdo en sostener la hipótesis de la desestructuración y la estandarización alimentaria sin introducir ciertos matices; entre otras razones porque, a raíz de los resultados de las investigaciones desarrolladas en las décadas de los años 80 y 90 , numerosos estudios han señalado la inconveniencia metodológica de hablar de desestructuración de las maneras de comer contemporáneas cuando no se disponen de encuestas diacró- 
nicas comparativas que puedan sostener tales supuestos. Por otro lado, se apunta que la desestructuración, en caso de existir, no afecta a todos los grupos de la misma forma ni con la misma intensidad. Son varios los investigadores cuyos trabajos plantean que la estandarización no es real y que no podemos hablar de una única alimentación como si todos los cambios habidos a lo largo de las últimas décadas hubieran suprimido las diferentes formas de comer según si uno es hombre o mujer, niño o adulto, campesino o industrial, rico o pobre. En contra de la tesis que plantea la disolución de las diferencias de clase, diversos autores, como Grignon (1980), Bourdieu (1988) o Gómez (1992), insisten en demostrar la permanencia del valor de la jerarquía social en el consumo alimentario contemporáneo, cuestionando las explicaciones psicologizantes que se han planteado en torno al comedor moderno y apuntando que las elecciones y las prácticas pueden siempre relacionarse con la clase social de pertenencia, en tanto que la abundancia y la democratización no han suprimido las desigualdades en el acceso a los recursos disponibles. En efecto, el componente de clase social, a pesar de que recientemente haya sido subsumido en distintos trabajos (Fischler 1995; Warde 1997) a otras variables tales como la edad o el género, continúa siendo un aspecto determinante de las elecciones alimentarias. No se puede obviar, por ejemplo, que en los países industrializados, durante las últimas décadas se ha venido incrementando las disparidades sociales en función del nivel de ingresos de las personas, de forma que los modelos de consumo de los más pobres permanecen iguales respecto a cuestiones históricamente definidas: están excluidos de la posibilidad de variedad y de calidad (Atkins y Bowler, 2001; Poulain, 2002a).

Aymard, Grignon y Sabban (1993:30) cuestionan abiertamente la tesis de la desestructuración alimentaria, estimando que, ante la ausencia de datos a los que poder acceder, ésta responde más bien a lo que se puede denominar "un rumor". Para estos tres investigadores dicha tesis, nacida a principios de los años 80 en el ámbito del marketing y retomada y popularizada por los sociólogos de la modernidad alimentaria, pretende descalificar la comida tradicional: "si la comida estructurada se encuentra así en receso; si es a la vez atacada desde el punto de vista de la modernidad, denunciada en nombre de la libertad individual, es quizá porque el modelo de comidas actualmente en uso en algunos países industrializados constituye efectivamente un obstáculo para el consumo extensivo y la alimentación continua con la que sueña la industria agroalimentaria, o, al menos, ciertas ramas de ésta como la bollería, la chocolatería, etc.".

En contra de las tesis que explican las tendencias desestructurantes como resultado de la modernidad alimentaria, Grignon (1993) argumenta que ésta no es más que la aplicación en el terreno particular de la alimentación de un escenario global de cambio derivado de las teorías del crecimiento que acompañan a la expansión y a las políticas económicas de los años 60; señala que, de hecho, lo que se produce es una especie de colonización de las hipótesis de la desestructuración de la alimentación moderna por los intereses agroindustriales. Grignon 
RIS

muestra que la situación en la sociedad industrial no es la de un cataclismo generalizado, en tanto que todavía las comidas principales de la población francesa incluyen las tres pautas principales correspondientes al desayuno, almuerzo y cena para una gran mayoría de franceses $(75,3 \%)$ y se hacen mayoritariamente en casa $(87,2 \%)$, lo que en realidad supone un freno al consumo extensivo o a la alimentación continua con la que, efectivamente, sueña la industria agroalimentaria, visiblemente interesada en ampliar al máximo la práctica del snacking o picoteo: comer en cualquier sitio y a cualquier hora. En el caso de España, los resultados del estudio presentado en el Foro Internacional de la Alimentación 2004 señalan en una dirección semejante: mientras que sólo el 11,8\% de las comidas se realizan fuera del domicilio, recurriendo para ello a la restauración privada y a comedores empresariales o escolares, la jornada alimentaria más común continúa componiéndose de tres ingestas (desayuno, comida y cena) o, máximo, de cuatro (añadiendo la merienda) (Contreras y Gracia, 2004).

Así pues, ¿cómo se ha de definir la "modernidad" alimentaria? ¿Por sus formas y grados de desestructuración, macdonalización, localismos o flexibilidad? Los estudios que se han realizado en Francia y otros países industrializados (Poulain 2002a; Contreras y Gracia, 2004) avalan parcialmente la tesis gastro-anómica propuesta por Fischler, en el sentido de que muestran una simplificación de la estructura de las comidas y un aumento de la importancia de la alimentación entre horas, a la vez que se pone en evidencia la existencia de un desfase entre las normas sociales relativas a las comidas y las prácticas realmente llevadas a cabo. Las primeras restituyen ampliamente la norma de la comida ternaria (entrada, plato compuesto y postres) y la secuencia ternaria (desayuno, comida y cena), así como la prohibición del picoteo, lo que explica una vez más que las verdaderas transformaciones en las maneras de comer actuales escapan en gran parte a las encuestas que utilizan únicamente los métodos declarativos $\mathrm{y}$, sobre todo, los métodos autoadministrados (De Garine, 1980; Calvo, 1980; Galán y Hercerbg, 1988 y 1999; Gracia, 1996b). Sin embargo, nos preguntamos hasta qué punto, de ser así, el modelo alimentario contemporáneo también deja un margen a las intenciones individuales y colectivas para restaurar, en el caso de que se haya perdido, el orden respecto a las prácticas y significados alimentarios.

En las sociedades industrializadas, con índices rápidos de crecimiento, el ejercicio de la elección entre una amplia serie de menús principales se convierte en algo plausible. Así, las personas podrían encontrar más factiblemente los alimentos para construir sus propias dietas personales haciendo opciones más o menos libres entre menús alternativos, seguramente en base al conjunto de circunstancias económicas y sociales que les rodean o del lugar donde el acto alimentario se produce. Beardsworth y Keil (1997) describen este contexto en términos de menus pluralism, es decir, de situaciones en las que varios esquemas alternativos son ofrecidos para estructurar las elecciones alimentarias y los modelos de comida. Este pluralismo es, en buena medida, producto de muchos 
procesos que se han combinado para dar paso al sistema alimentario moderno, como la industrialización de la producción y la distribución y la globalización del suministro de alimentos. Mientras que Fischler entiende todos estos procesos como causas que disgregan los referentes normativos de la sociedad gastro-nómica y aumentan la dispersión que llevan a la gastro-anomia, con todas las consecuencias negativas que ello acarrea, Beardworth y Keil son más optimistas al afirmar que, ciertamente, podría ser que las incertidumbres asociadas a la gastro-anomia no fueran más que síntomas de las tensiones individuales en la emergencia de un nuevo orden alimentario más abierto, flexible y plural.

Se trata de una sociedad, pues, en la que cabría todo: el particularismo cultural $\mathrm{y}$, al mismo tiempo, la globalización alimentaria; el individualismo $\mathrm{y}$, de forma paralela, nuevas formas de convivialidad alimentaria; finalmente, una sociedad de la afluencia y, a la vez, una sociedad que mantiene y aumenta la desigualdad en el acceso y la distribución de alimentos. Aunque los estudiosos no parecen ponerse de acuerdo respecto a la naturaleza de la modernidad alimentaria, sí se puede deducir de lo dicho hasta aquí que son las comidas, su transformación o su inmutabilidad, lo que está en el centro de este debate en tanto que elementos centrales del modelo alimentario. En cualquier caso, parece oportuno intentar caracterizarlas a partir de prolongar estas tesis, entendiendo que la mundialización de los mercados ha generado, al menos, tres movimientos - desaparición de ciertos particularismos locales, emergencia de nuevas formas alimentarias resultantes del proceso de mestizaje y difusión a escala transcultural de ciertos productos y prácticas alimentarias-y que todos ellos, de una manera $u$ otra, han redefinido las maneras de comer actuales.

Por su parte, los estudios que se han realizado en España teniendo en cuenta los criterios de desestructuración (Carrasco, 1992; González Turmo, 1995; Gracia, 1998; Kaplan y Carrasco, 1999) no han probado hasta ahora que exista una población pionera caracterizada por todos o muchos de los cuatro o cinco indicadores propios de la alimentación desestructurada, aunque la persistencia de la interiorización del modelo tripartito de las comidas, por un lado, y la constatación de otras maneras de comer alternativas que poco tienen que ver con ese modelo tradicional, por otro, invita a no descartar ninguna de las tesis propuestas.

En este sentido, el estudio realizado por el Observatorio de Alimentación en España constata una cierta individualización y simplificación de las comidas en diferentes sentidos (2004: 171): a) aumenta el número de comidas que se hacen en solitario; b) se amplían las franjas horarias de todas y cada una de las ingestas; c) se amplían y diversifican los lugares donde se realizan las comidas, tanto en el hogar (cocina, comedor, sofá, habitación, etc) como fuera de él (restauración pública y privada, plazas y jardines, transportes, calle, etc) y d) se produce una transferencia de las ingestas sólidas (relativamente concentradas en la comida y en la cena) en beneficio de las "pequeñas comidas" (el bocadillo o tentempié de la mañana, el aperitivo-comida, la merienda-cena o el "bocata" de la noche son las más frecuentes). Inversamente, el menú de las comidas principales se simplifica 
en su estructura: la comida principal al "nuevo estilo" se organiza menos en base al modelo ternario (primer plato, segundo plato y postres) y cada vez más alrededor de un solo plato. Tal concentración del menú sucede de forma más notoria durante las cenas. Siendo estas algunas de las características más notorias de las "nuevas" formas de comer en la sociedad española, la media de comida diarias se sitúa en 4,19, todavía muy lejos del promedio de los 20 food contacts registrados en EEUU, que señalan, para esta sociedad, un claro asentamiento del snacking.

Ello permite sostener que si bien las maneras de comer en España se han modificado y diversificado adaptándose a los nuevos constreñimientos impuestos por las transformaciones producidas en una sociedad cada vez más industrializada $y$, aun no correspondiéndose con el modelo alimentario facultativamente pautado y esperado, las prácticas observadas no constituyen, salvo excepciones, síntomas alarmantes de una desestructuración generalizada, sino más bien, asumiendo la propuesta de Beardsworth y Keil, modos de comer más operativos y flexibles.

\section{EL DESFASE ENTRE NORMAS Y PRACTICAS ALIMENTARIAS}

Aceptando, pues, la necesidad de caracterizar la compleja naturaleza de la modernidad alimentaria y aceptando también el interés por hacerlo a través de la articulación de las jornadas alimentarias, conviene ahora plantearse algunas cuestiones de carácter metodológico ¿Qué tipo de datos hay que recopilar? ¿Nos interesa lo que hacen realmente las personas? ¿O nos interesa aquello que dicen? ¿O ambas cosas a la vez? ¿Queremos analizar sus opiniones o actitudes, sus valores en relación con sus maneras de comer o en relación con productos alimentarios específicos? Todas estas preguntas son interesantes y permitirían llevar a cabo un análisis social de la modernidad alimentaria, pero no dan cuenta de las mismas cuestiones. Diferentes trabajos hechos desde una perspectiva socioantropológica indican que, efectivamente, hay un desfase significativo entre comportamientos alimentarios declarados y consumos reales (Fischler, 1995; Carrasco, 1992; Gracia, 1996; Corbeau y Poulain, 2002; Poulain, 2002b). Dichas diferencias se explican en buena medida a partir de comprender el papel que desempeñan, por un lado, las normas alimentarias y, por otro, las prácticas alimentarias. La mayor parte de nuestras conductas sociales individuales están articuladas por normas que nos indican lo que podemos o debemos hacer en cada momento y generan expectativas sobre lo que se considera deseable en un marco social más amplio. Otra cuestión diferente es que, como ya sabemos, las normas no siempre se cumplen o, en cualquier caso, se interpretan según conviene, dando paso a comportamientos más o menos distantes del sistema normativo.

El comer también está sometido a esta misma influencia y a este mismo proceso de enculturación/transgresión. De acuerdo con Poulain (2002b), nuestro comportamiento alimentario se articula mediante dos tipos de normas básicas: 
las normas sociales y las normas dietéticas ${ }^{1}$. Mientras que las primeras hacen referencia a las convenciones y normas que rigen la composición, combinación y estructura de las comidas, así como a las condiciones y contextos en los que se produce su consumo; las segundas incluyen el conjunto de prescripciones facultativas sobre qué alimentos son adecuados o no para la salud y están basadas en conocimientos científico-nutricionales y difundidas a través de medios médico-sanitarios. El aspecto más importante a considerar entre ambos tipos de normas es que se influyen mutuamente: a veces complementándose, a veces contradiciéndose. Los efectos de dicha relación están, a su vez, en la base de las prácticas alimentarias reales.

Fischler (1979), basándose en los resultados de estudios hechos sobre el comportamiento alimentario de la población estadounidense, señala una de las dificultades esenciales respecto a la recogida de datos sobre las prácticas cotidianas: "mientras que el número de tomas alimentarias (food contacts) en una misma jornada podía fácilmente llegar a ser de 20 , no obstante, los entrevistados declaraban mayoritariamente hacer 3 comidas al día, un poco como los amputados sienten su brazo o pierna perdida, como si fuera una especie de miembro fantasma". Ante la pregunta "¿cuántas veces comió ayer?", la respuesta espontánea remite a las normas interiorizadas, que pueden ser tanto las sociales como las dietéticas: 3 en lugar de 20. Lo mismo sucede cuando se interroga en estos términos a una persona sobre aquello que ha comido: "qué y con qué frecuencia comió usted ayer, en la última semana o en el último mes tales productos" y responde "un poco de todo, verduras, carnes, pescados, lácteos, cereales...". Nuevamente, la respuesta es estereotipada: nos remite a un modelo de consumo "equilibrado", mientras que hace referencia sólo a alimentos y no a platos, a productos aislados, sin aludir a su manipulación y ubicación en el contexto más amplio del consumo alimentario. Esta respuesta, este desfase tan extraordinario, constituye un objeto de estudio privilegiado para analizar las representaciones sociales en torno a las comidas. Sin embargo, revela un problema de orden metodológico para aquellos estudios cuyo objetivo principal consista en saber qué, cuándo, con quien, dónde y cómo come la gente, es decir, cuáles son sus prácticas alimentarias reales.

\footnotetext{
'Algunos ejemplos de normas sociales alimentarias serían: distribuir la comida a lo largo del día en una toma o varias; establecer unos horarios definidos según el tipo de comidas; considerar unas ingestas más importantes que otras; adoptar unos modales particulares a la hora de comer respecto a nuestra apariencia fisica; comenzar nuestras comidas con alimentos salados en lugar de dulces; comer dependiendo de los platos y alimentos con los dedos o con cubiertos; establecer un calendario alimentario según las ocasiones festivas, etc. Ejemplos de normas dietéticas podrían considerarse: comer abundantes frutas, verduras o cereales; consumir pescado azul, alimentos con fibra o aceite de oliva; utilizar técnicas para tratar y cocinar los alimentos evitando la pérdida de nutrientes básicos; evitar el consumo de grasas animales saturadas o de azúcares simples; ajustar la ingesta calórica a las necesidades energéticas; comer despacio y masticar los alimentos, etc.
} 
RIS

REVISTA INTERNACIONAL DE SOCIOLOGÍA

№ 40, ENERO-ABRIL, 2005

Los referentes normativos también afectan a la categorización de las comidas. En España, como en otros países de Europa, la comida principal se entiende como una unidad constituida por tres categorías: primer plato, segundo plato y postres. Una versión más compleja (de cuatro o cinco categorías) nos remite a las ocasiones especiales o celebraciones (fiestas navideñas, menú dominical, aniversarios...), las cuales se convierten en festivas precisamente por la variación que se registra respecto a una estructura más simple: aperitivos, entrantes, dulces, etc. Podemos afirmar que el primer tipo de estructura es el considerado más habitual: lo encontramos en los menús escolares, en los centros hospitalarios, en los comedores de empresa, en la restauración, en las propuestas dietético-culinarias de la prensa escrita y de numerosos libros; en nuestras casas también. Esta estructura ternaria constituye, en términos de prácticas declaradas, "la comida más normal". Ahora bien, ¿hasta qué punto dicha estructura es la más frecuente en las diversas "comidas normales" que hacemos a lo largo del día? Por otro lado, ¿son éstas las más representativas estadísticamente hablando?

Teniendo en cuenta todas estas premisas, convendría preguntarse cómo caracterizar las maneras de comer contemporáneas. De acuerdo con Poulain (2002b), primero debemos distinguir entre qué son las prácticas reales (observadas) y las prácticas declaradas (interiorizadas). Estas últimas son las que hacen declarar a las personas ante ciertas preguntas que gastan veinte euros en lugar de diez, que siempre compran pescado fresco en lugar de congelado o que cuando llenan el cesto de la compra lo hacen siempre pensando más en los gustos de los demás que en los suyos propios. Por ejemplo, en raras ocasiones las personas responsables de la alimentación familiar admiten variar, por razones de orden presupuestario, sus prácticas alimentarias, especialmente el tipo o la calidad de los alimentos consumidos, cuando sabemos positivamente que de todas las partidas del presupuesto familiar la alimentaria, aún siendo fija, es la más flexible. Sin embargo, en la valoración del gasto alimentario se incluyen valores de carácter moral. Reconocer que se reduce el presupuesto destinado a la alimentación en beneficio de partidas como el ocio o la cosmética equivale a reconocer, directa o indirectamente, que se escatima en salud y en el cuidado de los miembros del grupo doméstico (Gracia, 1996b).

Comparando unas con otras, las prácticas declaradas muestran que son fácilmente objeto de transformación, de reestructuración semántica, de olvido o, aún más, de negociación o ocultación. Ante estas circunstancias, lo primero que debemos plantearnos es si existe algún tipo de especificidad en relación con el comportamiento alimentario que potencie, más que en otros ámbitos, el desajuste entre el discurso mantenido por los informantes y sus prácticas reales. La respuesta parece ser, a todas luces, afirmativa. Las diferencias semánticas que pueden registrarse, sin ir más lejos, a la hora de definir términos tan utilizados por todo el mundo como alimentación, comida o alimento, están estrechamente vinculadas a la misma dificultad expresada por los informantes al referir su significado y per- 
cibir las connotaciones atribuidas a cada uno de estos términos. La significación que articula el hecho alimentario es compleja además de cambiante, y entre los diferentes aspectos que lo definen son los condicionantes económicos, los valores éticos y morales, la identidad cultural o la jerarquización social los que se erigen en constreñimientos materiales y simbólicos que potencian dicho desajuste.

La alimentación cotidiana, participando de la esfera tanto íntima como pública de las personas, es, a menudo, transformada por la oralidad y por los discursos que construyen los informantes, de tal manera que se hace necesario recoger la información utilizando recursos técnicos que permitan la contrastación. A la etnografía le interesa ver cómo se construye dicha oralidad y no sólo porque a través de ella se expresa el punto de vista interno de los actores sociales o emic, extraordinariamente útil en la tarea de comprender lo que es verdaderamente significativo para el grupo estudiado, sino porque su relato da cuenta de cómo los actores sociales organizan el conocimiento y sus experiencias alimentarias a través del lenguaje, cómo las perciben y cómo las comunican. Pero también le interesa conocer, mediante técnicas que favorezcan la observación y la descripción, cómo se materializan tales experiencias.

La constatación empírica de este desfase no es, por otro lado, difícil. Lo importante es reconocer su existencia para abordarlo analíticamente. El estudio realizado por el Observatorio de la Alimentación en España (2004) nos da cuenta de las diferencias referidas. Sirva de ejemplo, la conceptualización de lo que se entiende por un "verdadero desayuno" y los alimentos que finalmente son consumidos durante esta ocasión. Leche, cereales, tostada con aceite, fruta cruda o en zumo, son los ingredientes que se consideran ideales para un buen desayuno:

"Para mí, tiene que ser zumo, cereales, y fruta, pero...".

"Para mí sería lo ideal leche, cereales, tostada con aceite, fruta...".

"Por la mañana me gustaría desayunar bien... una tostada, un huevo, frutas o zumos naturales...".

Sin embargo, en el desayuno que en realidad se ingiere, los alimentos más habituales son los comprendidos dentro de la categoría de "bollería, galletas y dulces" $(45,6 \%)$. En un segundo nivel, se sitúan el pan y las tostadas $(18,9 \%)$, siguen los cereales $(17,4 \%)$ y los aceites y mantequillas $(17,3 \%)$. En menor medida, pero con un porcentaje significativo, aparece también el consumo de embutidos y quesos (en un $8,6 \%$ de los desayunos). Como puede observarse, a pesar de las recomendaciones dietéticas relativas a la conveniencia de incluir las frutas en el desayuno y a pesar, también, de que las mayoría de la población considera que un "verdadero desayuno" debe incluir las frutas, sólo en un 4,7\% de los casos se han incluido las frutas en el desayuno. En esta ingesta, las dos bebidas más habituales son el café con leche $(38,4 \%)$ y la leche y otros lácteos $(37,2 \%)$. Otras bebidas 
consumidas con bastante frecuencia son el café $(15,1 \%)$ y los zumos $(8,4 \%)$ Otra vez, resulta significativo, a causa de la discrepancia entre lo considerado como un verdadero desayuno y lo realmente consumido, el bajo porcentaje que representan los zumos.

\section{CONCLUSIONES}

Desde un punto de vista teórico y metodológico convendría dejar claro, volviendo así a nuestro punto de partida, que aunque parece que las normas interiorizadas por la mayoría de la población testimonian un buen nivel de apropiación de los discursos nutricionales fruto de la progresiva medicalización del comportamiento cotidiano, las prácticas alimentarias siguen motivadas por un conjunto de constreñimientos materiales y simbólicos, de tal forma que no parece existir una correspondencia directa entre, por una parte, las recomendaciones dietéticas asumidas por las personas y su definición de dieta saludable y, por otra, los consumos reales. Así, pues, tales resultados ponen de manifiesto, nuevamente, que la salud no es la única motivación para alimentarse ni para hacerlo de un modo determinado, y que la alimentación es funcional en cada una de sus circunstancias o contextos. Además de la nutricional, cabe considerar otras funciones importantes de la alimentación que tienen que ver con las necesidades más inmediatas y cotidianas y con las fórmulas más prácticas o posibles para resolverlas. Razones relativas a otros imperativos circunstanciales (disposición del tiempo y presupuesto, tipos y grados de conveniencia, búsqueda de placer o de sociabilidad) condicionan y determinan del mismo modo, o incluso más, las maneras de comer.

La complejidad asociada a la naturaleza de la modernidad alimentaria convierte en cuestionables o, cuando menos, en insuficientes aquellas aproximaciones metodológicas que únicamente contemplan la aplicación de cuestionarios que favorecen respuestas ideales fruto de la interacción entre normas sociales y normas dietéticas, a menudo muy estereotipadas y que, por consiguiente, no abordan el desfase existente entre las normas alimentarias socialmente aceptadas y las actitudes de la población $\mathrm{y}$, de forma particular, entre las prácticas declaradas y las prácticas observadas. Hay que rescatar desde la observación directa este espacio de significación, en tanto que es la única vía posible para poder caracterizar y matizar las maneras de comer contemporáneas: de qué dependen, cómo se articulan, qué y quiénes contribuyen a su modificación. No es de extrañar que mientras desde las instancias políticas y sanitarias los comportamientos alimentarios sigan siendo sólo entendidos como meros hábitos (en el sentido de repetición mecánica de ciertos actos racionales cuya modificación tendría que ser a priori sencilla), la puesta en marcha de programas de educación nutricional, las recomendaciones dietéticas o los intentos por cambiar ciertas conductas caigan a menudo en "saco roto" o acumulen tantos fracasos, aunque ello pueda parecer incluso paradójico en una sociedad que se caracteriza por la medicalización de un sinfín de procesos cotidianos. 
Por otro lado, quienes aconsejan, intervienen o recomiendan, necesitan saber cuáles son las prácticas reales, a menudo heterogéneas y complejas, para saber si hay que mejorarlas o no. Que el modelo alimentario "tradicional" haya experimentado transformaciones en diferentes direcciones no debe interpretarse exclusivamente como un empeoramiento de la salud de la población producto de la progresiva desestructuración de sus hábitos alimentarios. Asimismo, los prescriptores necesitan valorar hasta qué punto sus "recomendaciones" son más o menos compatibles con otros constreñimientos tanto o más importantes que afectan elecciones alimentarias de las personas, al margen de que éstos puedan poner o no a ciertos grupos sociales en situaciones de "riesgo" para su salud. Sea como fuere, debemos considerar las prácticas supuestamente de riesgo para la salud como aspectos de la vida cultural $\mathrm{y}$, en consecuencia, determinadas también por factores socioculturales. Hay que evitar también las generalizaciones y los alarmismos oportunistas, especialmente teniendo en cuenta que las "nuevas" maneras de comer contemporáneas coinciden, en las sociedades industrializadas, con una mejora de la calidad de vida y del estado de salud de la población: nunca como ahora los alimentos habían sido tan abundantes, los controles de calidad dentro de la cadena alimentaria tan numerosos y efectivos y nunca, tampoco, la esperanza de vida de las personas había alcanzado niveles tan altos. Ello hace pensar que, efectivamente, no estamos en una situación alimentaria ni tan desestructurada ni tan caótica.

\section{REFERENCIAS BIBLIOGRÁFICAS}

ADAM, P. y C. HERZILCH (1994), Sociologie de la maladie et de la médicine, París, Nathan.

ARIÈS, P. (1997a), La fin de mangeurs, Paris, Desclée de Brouwer.

(1997b), Les fils de McDo. La McDonalisation du monde, París, L'Harmattan.

ATKINS, P. e I. BOWLER (2001), Food in society. Economy, culture, geography, Nueva York, Arnold.

AYMARD, M, C. GRIGNON, y F. SABBAN (1993), Le temps de manger, alimentation, emploi $d u$ temps et rythmes sociaux, Paris, Éditions MSH-INRA.

BAUMAN, Z. (2001), La postmodernidad y sus descontentos, Madrid, Akal.

BEARDSWORTH, A. y T. KEIL (1997), Sociology on the menu. An invitation to the study of food and society, Londres, Routledge.

BECK, U. (2001), La sociedad del riesgo, Hacia una nueva modernidad, Barcelona, Piados.

(2002), La sociedad del riesgo global, Madrid, Siglo XXI.

BOURDIEU, P. (1988), La distinción. Criterios y bases sociales del gusto, Madrid, Taurus. 
RIS

REVISTA INTERNACIONAL DE SOCIOLOGIA

N० 40, ENERO-ABRIL, 2005

MABEL GRACIA ARNAIZ

CALVO, M. (1980), "De la contribution actualle des Sciences Sociales à la connaissance de l'alimentation", Ethnologie Française, Vol. 10 (3), pp. 355-352.

CARRASCO, S. (1992), Antropologia i alimentació. Una proposta per a l'estudi de la cultura alimentaria, Bellaterra, Servei de Publicacions U.A.B.

CHEYNE, G. (1733), The English Malady, Londres, Strachan y J. Leake.

CONRAD, P. (1992), "Medicalization and social control", Annual Review of Sociology, Vol. 18, pp. 209-232.

CORBEAU, J.P. (1992), "Rituels alimentaires et mutations socials", Cahiers internationaux de sociologie, Vol. XCII, pp.

CORBEAU, J.P. y J.P. POULAIN (2002), Penser l'alimentation. Entre imaginaire et rationalité, Paris, Éditions Privat.

CONTRERAS, J. (1999), "Cambios sociales y cambios en los comportamientos alimentarios en la España de la segunda mitad del siglo XX”, Anuario de Psicología, Vol. 30, (2), pp. 25-42.

CONTRERAS, J. y M. GRACIA (dirs), (2004), La alimentación y sus circunstancias: placer, conveniencia y salud, Barcelona, IV Foro Internacional de la Alimentación.

COVENEY, J. (1999), "The Government of the table: nutrition expertise and the social organisation of family food habits", en J. Germov y L. Williams (eds), A sociology of food and nutrition, Oxford, Oxford University Press.

CRAWFFORD, R. (1997), "You are dangerous to your health, the ideology of victim blaming", International Journal of Health Services, Vol. 7, (4), pp. 663-680.

DE GARINE, I. (1980), “Une anthropologie alimentaire des Français?", Ethonologie Française, vol. 10, (3), pp. 227-238.

(2002), "Antropología de la alimentación y autenticidad cultural”, en M. Gracia (coord.), Somos lo que comemos. Estudios de alimentación y cultura en España, Barcelona, Ariel.

DOUGLAS, M. (1979), «Les estructures du culinaire», Communications, $\mathrm{n}^{\circ} 31$ (versión en castellano en J. Contreras (comp), Alimentación y cultura. Necesidades, gustos y costumbres, Barecelona, Universidad de Barcelona, 1995).

FISCHLER, C. (1979), “Gastro-nomie et gastro-anomie”, Communications, nº 31, pp. 189-210.

(1990), L'(H)omnivore, París, Odile Jacob (la versión en castellano ha sido publicada por Anagrama, 1995, Barcelona).

(1996), "La macdonalisation des moeurs", en J.L. Flandrin y M. Montanari (eds.), Histoire de l'alimentation, París, Fayard.

IV FORO ALIMENTARIA INTERNACIONAL DE LAALIMENTACIÓN (2002), ¿Adónde va la alimentación?, Barcelona. 
GALÁN, P. y S. HERCERBG (1988), "Las encuestas alimentarias: utilización en los estudios epidemiológicos de tipo nutricional", en S. Hercerbg et al. (coords.), Nutrición y Salud Pública. Abordaje epidemiológico y políticas de prevención, Madrid, CEA.

(1999), "Méthodes de mesure de la consommations alimentaire", Cahiers de nutrition et de dietétique, vol.34, pp. 50-54.

GIDDENS, A. (1991), Modernity and Self-Identity, Cambridge, Polity Press.

GIDDENS, A. et al. (1996), Las consecuencias perversas de la modernidad, Barcelona, Anthropos.

GÓMEZ, F. (1992), “Gastro-anomie: apparence ou réalité?”, Cahiers internatinaux de psychologie sociale, $\mathrm{n}^{\circ} 13$, pp. 60-64.

GONZÁLEZ TURMO, I. (1995), Comida de rico, comida de pobre. Los hábitos alimenticios en el Occidente andaluz (siglo XX), Sevilla, Universidad de Sevilla.

GRACIA, M. (1996a), Paradojas de la alimentación contemporánea, Barcelona, Icaria.

(1996b), "El decalage entre el discurso del informante y sus prácticas: el caso de la alimentación", en J. L. García (coord.), Etnolingüistica y análisis del discurso, Vol. II, Actas del VII Congreso de Antropología Social, Zaragoza.

(1998), La transformación de la cultura alimentaria. Cambios y permanencias en un contexto urbano (Barcelona, 1960-1990), Madrid, Ministerio de Cultura.

GRIGNON, C. (1993), "La règle, la mode et le travail", en M. Aymard, C. Grignon y F. Sabban (eds.), Le temps de manger. Alimentation, emploi du temps et rythmes sociaux, París, MSH-INRA.

GRIGNON, C. y Ch. GRIGNON (1980a), "Styles d'alimentation et goûts populaires", Revue Française de Sociologie, París.

(1980b), Consommations alimentaires et styles de vie, París, INRA.

HERBERT, V.(1990), "The Mount Sinai School of Medicine. Complete Book of Nutrition", Subak-Sharpe.

HERPIN, N. (1980), “Comportaments alimentaires et contraintes sur l'emploi de temps", Revue Française de Sociologie, vol. 21, pp.

HERPIN, N. y D. VERGER (1991), La consommation des Français, París, La Découverte.

KAPLAN, A. y S. CARRASCO (1999), Migración, cultura y alimentación. Cambios y continuidades en la organización alimentaria, de Gambia a Cataluña, Bellaterra, UAB.

LEWIN, K. (1943), "Forces behind food habits and methods of change", en Committee of Foods Habits, The problem of changing food habits, Natl. Res. Counc. Bull. Washington DC.

MAPYA (series históricas 1991-2002), La alimentación en España, Madrid, Ministerio de Agricultura, Pesca y Alimentación. 
RIS

REVISTA INTERNACIONAL DE SOCIOLOCIA

№ 40, ENERO-ABRIL, 2005

MABEL GRACIA ARNAIZ

MEAD, M. (1943), "Dietary patterns and food habits", Journal of the American Dietetic Association, vol 19, (1), pp.1-5.

MENNELL, S. (1985), All manners of food. Eating and Taste in England and France from de Middle Age to the Present, Oxford, Basil Blackwell.

POULAIN, J. P. (2002a), Sociologies de l'alimentation, París, P.U.F.

(2002b), Manger aujourd'hui. Attitudes, normes et pratiques, Toulouse, Éditions Privat.

RITZER, G. (1992), The McDonalization of Society, Londres, Pine Forge Press.

(2001), Explorations in the sociology of consumption. Fast food, credit cards and casinos Londres, SAGE.

RICHARDS, A. (1932), Hunger and work in a savage tribe: a functional study of nutrition among de southerm bantu, Londres, Routledge.

(1939), Land, labour and diet in Northern Rhodesia, Londres, Oxford University Press.

RYAN, W. (1977), Blaming the victim, Nueva York, Vintage Books.

TANNAHILL, R. (1988), Food in History, Londres, Penguin Books.

THOMAS, J. (1980), "The relationship between knowledge about food and nutrition and food choice", en M. R. Turner (ed), Nutrition and lifestyles, Londres, Applied Science Publishers.

TURNER, B. S. (1999), "The discourse of diet", en M. Featherstone, M. Hepworth y B. Turner (eds.), The body. Social Process and Cultural Theory, Londres, SAGE Publications.

VEBLEN, Th. (1899), The Theory of Leisure Class, Nueva York, Mentor.

WARDE, A. (1997), Consumption, food and taste: culinary antinomies and commodity of the consumer, Londres, Sage Publications. 